\title{
Esthetic Management of Gingival Melanin Hyperpigmentation by 810 nm Diode Laser
}

\section{Munishwar S $^{1 *}$, Manab $\mathrm{K}^{2}$ and Mohinder $\mathbf{P}^{2}$}

${ }^{1}$ Department of Periodontology, 201 Military Dental Centre, India

${ }^{2}$ Department of Periodontology, Department of Dental \& Oral Health Sciences, Armed Forces

Medical College, India

*Corresponding author: Munishwar Singh, Department of Periodontology, 201 Military

Dental Centre, C/o 99 APO, India, 981027, Email: msmalhi@yahoo.com

\section{Case Report}

Volume 3 Issue 4

Received Date: September 14, 2018

Published Date: October 01, 2018

DOI: 10.23880 /oajds-16000196

\section{Abstract}

Gingival hyperpigmentation and excessive gingival display are the main esthetic concerns of a large number of patients visiting a dentist. The color of gingiva is determined by the vascular supply, the thickness of epithelium, its degree of keratinization and the presence of melanin granules which are produced in melanosomes of melanocytes. Melanocytes are located in basal and supra basal layers of the epithelium and are responsible for the melanin pigmentation of the gingiva. Gingival hyperpigmentation can occur irrespective of age or sex and can vary from linear/ diffuse/ triangular patches of light brown to dark brown or bluish black color. Melanin pigmentation is a physiological pigmentation and no treatment is required for same but in the present cosmetic and esthetic era, a number of patients are conscious of the dark patches of pigmentation and request for its treatment. Among a plethora of available treatment modalities for this condition, laser has shown to be the most promising solution. This case report highlights the effectiveness of $810 \mathrm{~nm}$ diode laser in treating gingival hyperpigmentation.

Keywords: Melanin; Physiologic pigmentation; Gingiva; Depigmentation; Diode laser

\section{Introduction}

Melanin, a non-hemoglobin derived brown pigment is produced by melanocytes which are located in basal and supra basal layers of epithelium. It is responsible for the pigmentation of skin, gingiva and oral mucous membrane [1]. This physiological pigmentation is genetically determined and also influenced by other factors like ultraviolet radiation, activity of the endocrine glands, smoking and medication. The gingival color is affected by the number and size of vasculature, the epithelial thickness and its degree of keratinization along with the pigments present within the gingival epithelium [1]. The primary pigments contributing to normal color of oral mucosa are melanin, melanoid, carotene, hemoglobin, hemosiderin and bilirubin. Melanin pigmentation is the most common pigmentation seen in the oral soft tissues and is the common cause of gingival hyperpigmentation [2].
This physiologic pigmentation requires no treatment, but is a cause of concern for individuals with a high consideration for esthetics or with a high smile line.

Gingival depigmentation is a treatment modality to remove the melanin hyper pigmentation and there are various techniques to address this esthetic problem (Table 1). Diode lasers have various wavelengths and have been commonly used to treat gingival depigmentation with no side effects. Diode Lasers rely on their affinity for colored pigments like melanin and their ablative property targets the melanin present in melanocytes of basal and suprabasal layers of epithelium that absorb and convert light energy into heat by photo thermolysis [3]. This case report highlights a case of gingival pigmentation treated successfully by $810 \mathrm{~nm}$ diode laser. 
1. Methods aimed at eliminating gingival pigmentation

a. Surgical Methods

$\begin{array}{ll}\text { i. } & \text { Scalpel surgical technique } \\ \text { ii. } & \text { Electrosurgery } \\ \text { iii. } & \text { Cryosurgery } \\ \text { iv. } & \text { Abrasion } \\ \text { v. } & \text { Lasers }\end{array}$

- Argon

- Gallium aluminium arsenide (GaAlAs)

- Indium Gallium arsenide (InGaAs)

- Neodymium-doped:yttrium, aluminium and garnet (Nd:YAG)

- Erbium, chromium-doped yttrium, scandium, gallium, and garnet (Er,Cr:YSGG)

- Erbium-doped: yttrium, aluminium and garnet (Er:YAG)

- Carbon dioxide $\left(\mathrm{CO}_{2}\right)$

b. Chemical Methods (not used these days)

i. $\quad 90 \%$ phenol in combination with $95 \%$ alcohol

ii. $5 \%$ paraformaldehyde

iii. Potassium hydroxide

iv. Other chemicals

2. Methods aimed at masking the gingival pigmentation

a. Free Gingival Grafts

b. Acellular dermal matrix allografts

c. Connective Tissue Graft

Table 1: Different gingival depigmentation methods.

\section{Case Report}

A 22-year-old patient reported to the dental department with a chief complaint of "darkened gums" that affected his self-confidence. The patient requested for any cosmetic therapy that would enhance his smile and esthetics. On general examination, the patient was well oriented to time, place and person and no abnormality was detected. He was healthy and not under any medication. On clinical examination, the periodontal tissues were healthy but bilateral melanin pigmentation was present in both maxilla and mandible (Figure 1). The Patient scored a melanin pigmentation score of "4" on Dummet-Gupta oral pigmentation index [4] (1: no clinical pigmentation, 2: mild clinical pigmentation, 3: moderate clinical pigmentation, and 4: heavy clinical pigmentation). The depigmentation procedure was verbally explained to the patient and a written informed consent was obtained. All the laser safety precautions were strictly adhered to during the procedure. Topical anesthesia was given before each laser raster and an $810 \mathrm{~nm}$ diode laser
(ZOLAR Photon, Zolar Technology \& Manufacturing Co. Inc, Canada) was used for the bilateral depigmentation of the maxillary and mandibular anterior gingiva up to the mesial aspect of first premolars. The fiber-optic laser tip at 1.0 to $1.2 \mathrm{~W}$ power in continuous wave mode was used. A light contact to very light brush stroke like movement of the beam through the tissue was employed, while taking care to avoid injury to tooth surfaces or adjacent tissues. The depigmented area was then wiped with a gauze soaked in saline (Figures 2 \& 3). After the completion of the procedure, no periodontal dressing or antibiotics were prescribed. Analgesic $(500 \mathrm{mg}$ Paracetamol) was advised incase if the need arose. Patient was instructed to avoid hot, acidic and spicy food for 4-5 days as that could disturb the healing process and cause discomfort. Patient was recalled at 1 and 4 weeks during the post-operative period for clinical evaluation. The healing was uneventful and the gingiva appeared healthy pink which scored a score of "1" on Dummet-Gupta oral pigmentation index (Figure 4). 


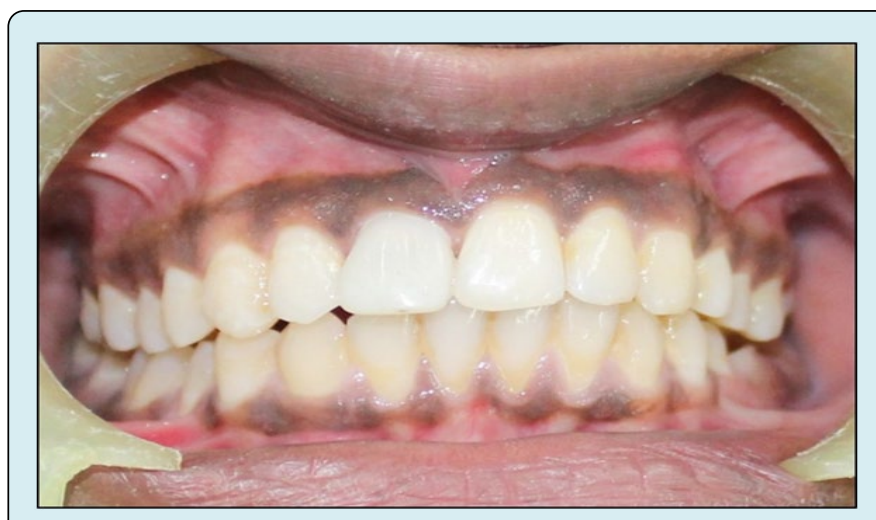

Figure 1: Pre-operative Evaluation.

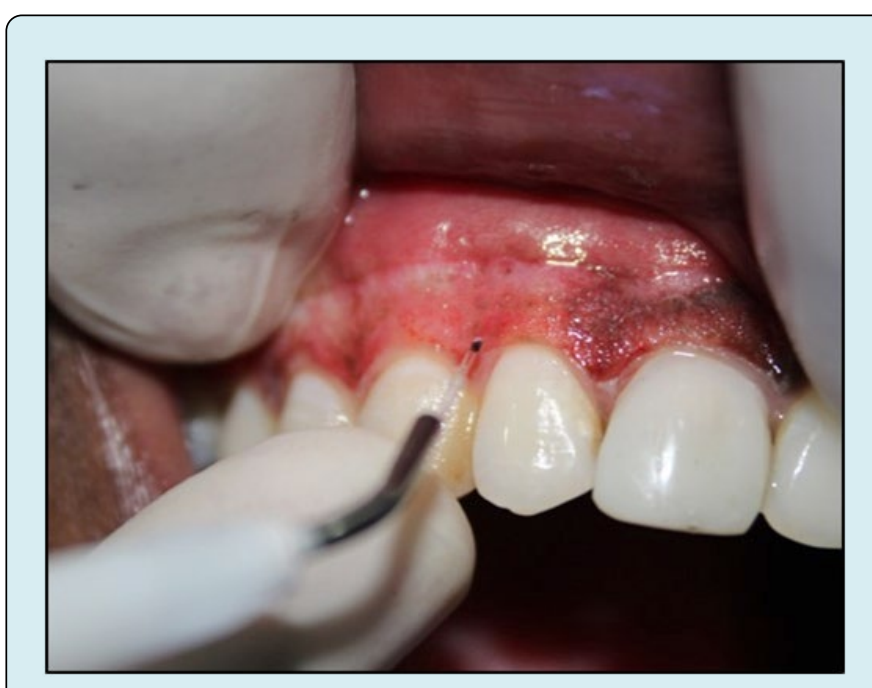

Figure 2: Application of $810 \mathrm{~nm}$ Diode laser in maxillary arch.

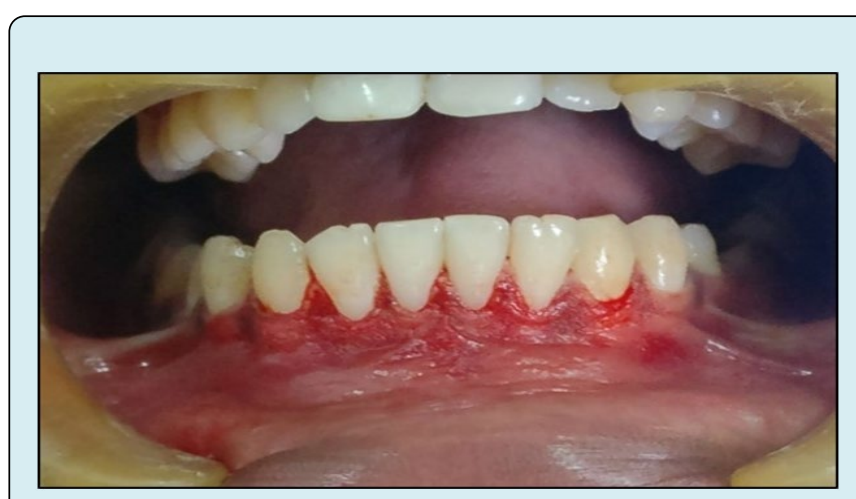

Figure 3: Completion of gingival depigmentation in mandibular arch.

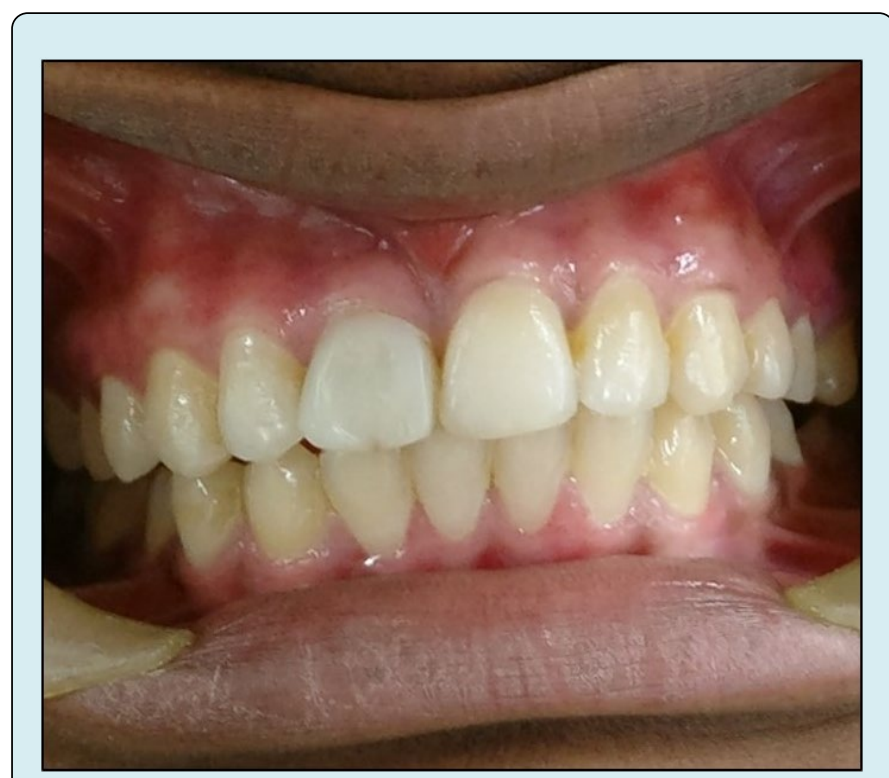

Figure 4: 01-month Post-op evaluation.

\section{Discussion}

In the present era of heightened demand for esthetics, patients reporting for gingival depigmentation have increased. Scalpel surgical technique has been a gold standard and a treatment of choice for gingival depigmentation but over the last decade laser has gained wide acceptance both with the clinicians and patients alike. Lasers are an effective, pleasant and reliable treatment modality with minimal post-operative discomfort. The lasers have shown to result in homogenous ablation of epithelial and rete pegs along with having a bactericidal effect and better hemostasis [5]. $810 \mathrm{~nm}$ diode laser is a Gallium aluminium arsenide (GaAlAs) laser which has a high affinity for colored pigments like hemoglobin and melanin thus making it a preferred laser for gingival depigmentation procedures [6]. The use of lasers has many advantages such as less demand for anesthesia, no requirement for placement of periodontal dressing, short chair side time, no or very slight pain, no hemorrhage and better patient acceptance. The only disadvantage of lasers is the cost associated with the laser machine.

The diode laser ensured a clear and dry operating field thus less chair side time. The reduced pain perception with laser depigmentation in comparison to scalpel technique could be attributed to the protein coagulum formed on the surface of ablated surface which acts as a biologic dressing in addition to the sealing of ends of sensory nerves [7]. 
The success of depigmentation by any modality is weighted by the time and extent it takes for the repigmentation to re-appear after the treatment. The mechanism of re-pigmentation is not clearly understood but as per migration theory, active melanocytes from adjacent pigmented tissues migrate to the treated area, causing repigmentation [8].

According to a systematic review by Lin, et al. [9] repigmentation was reported with nearly all the depigmentation methods and lasers showed the least recurrence rates when compared with bur abrasion, scalpel surgery, and some other methods. During the follow-up of this patient, no re-pigmentation has been observed till date and the patient is on a regular follow up.

\section{Conclusion}

The use of diode laser found more acceptance with the patient in comparison to surgical scalpel technique and he was pleased with the outcome of the result. This case study reveals that depigmentation with an $810 \mathrm{~nm}$ is a reliable, safe, effective and better treatment modality for gingival depigmentation.

Conflicts of Interest: There are no conflicts of interest.

\section{References}

1. Carranza F, Newman M, Takei H, Klokkevold P (2012) Carranza's Clinical Periodontology. $11^{\text {th }}$ (Edn.), St. Louis, Mo: Elsevier Saunders.

2. Dummett CO, Barens G (1967) Pigmentation of the Oral Tissues: A Review of the Literature. J Periodontol 38(5):
369-378.

3. Basha MI, Hegde RV, Sumanth S, Sayyed S, Tiwari A, et al. (2015) Comparison of Nd: YAG laser and surgical stripping for treatment of gingival hyperpigmentation: a clinical trial. Photomed Laser Surg 33(8): 424436.

4. Dummet CO (1946) Physiologic pigmentation of the oral and cutaneous tissues in the negro. J Dent Res 25(6): 421-432.

5. El Shenawy HM, Nasry SA, Zaky AA, Quriba MA (2015) Treatment of gingival by diode laser for esthetical purposes. Maced J Med Sci 3(3): 447-454.

6. El Shenawy HM, Fahd A, Ellabban M, Dahaba M, Khalifa $M$ (2017) Lasers for esthetic removal of gingival hyperpigmentation: A systematic review of randomized clinical trails. Int J Adv Res 5(3): 1238-1248.

7. Moritz A, Schoop U, Strassl M, Wintner E (2006) Lasers in Endodontics. In: Moritz A (Ed.), Oral Laser Application. Berlin: Quintessenz, pp: 100.

8. Grover HS, Dadlani H, Bhardwaj A, Yadav A, Lal S (2014) Evaluation of patient response and recurrence of pigmentation following gingival depigmentation using laser and scalpel technique: a clinical study. J Indian Soc Periodontol 18(5): 586-592.

9. Lin YH, Tu YK, Lu CT, Chung WC, Huang CF, et al. (2014) Systematic review of treatment modalities for gingival depigmentation: a randomeffects Poisson regression analysis. J Esthet Restor Dent 26(3): 162178.

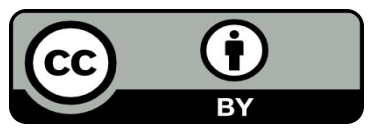

